\title{
CAYLEY PROPERTIES OF MERGED JOHNSON GRAPHS
}

\author{
GARETH A. JONES AND ROBERT JAJCAY
}

\begin{abstract}
Extending earlier results of Godsil and of Dobson and Malnič on Johnson graphs, we characterise those merged Johnson graphs $J=J(n, k)_{I}$ which are Cayley graphs, that is, which are connected and have a group of automorphisms acting regularly on the vertices. We also characterise the merged Johnson graphs which are not Cayley graphs but which have a transitive group of automorphisms with vertex-stabilisers of order 2. Even though these merged Johnson graphs are all vertex-transitive, we show that only relatively few of them are Cayley graphs or have a transitive group of automorphisms with vertex-stabilisers of order 2.
\end{abstract}

MSC classifications: 05E18 (primary), 20B05, 20B20, 20B25 (secondary).

Key words: Johnson graph, Cayley graph, $k$-homogeneous group.

\section{INTRODUCTION}

There is a folklore conjecture that, up to a given sufficiently large order, most connected vertex-transitive graphs are Cayley graphs, or equivalently, by a result of Sabidussi [24], that most such graphs have a group of automorphisms acting regularly on the vertices. Censuses of graphs of small order, such as that by McKay and Praeger in [22], or that of cubic graphs by Potočnik, Spiga and Verret in [23], provide evidence to support this conjecture. However, if one restricts attention to certain particular families of vertextransitive graphs, the picture can be very different.

The smallest vertex-transitive graph which is not a Cayley graph is the Petersen graph. This is the odd graph $O_{3}$, or equivalently the Kneser graph $K(5,2)$. More generally, the Kneser graph $K(n, k)$ has the set $\left(\begin{array}{l}N \\ k\end{array}\right)$ of $k$-element subsets of an $n$-element set $N$ as its vertices, adjacent if and only if they are disjoint; the action of the symmetric group $S_{n}$ on $N$ shows that Kneser graphs are vertex-transitive. Taking $n=2 k+1$ gives the odd graph $O_{k+1}:=K(2 k+1, k)$. Answering a question of Biggs [3] about whether any odd graphs are Cayley graphs, Godsil showed in [13] that $K(n, k)$ is not a Cayley graph unless either $k=2, n$ is a prime power and $n \equiv 3 \bmod (4)$, or $k=3$ and $n=8$ or 32 .

Godsil's proof uses the fact that $S_{n}$ is the full automorphism group of $K(n, k)$, so a group of automorphisms acts vertex-transitively on this graph if and only if it is a $k$-homogeneous permutation group of degree $n$ (one which is transitive on $k$-element subsets), and it acts 
regularly if and only if it is sharply $k$-homogeneous (regular on $k$-element subsets). Results of Kantor [19] and of Livingstone and Wagner [20], summarised here in Theorem 3.1, provide a complete description of the latter groups (see cases (1), (2) and (3) of Theorem 1.1), so the result follows.

The Johnson graph $J(n, k)$ has the same vertex set $\left(\begin{array}{c}N \\ k\end{array}\right)$ as $K(n, k)$, but in this case two $k$-element subsets are adjacent if and only if their intersection has $k-1$ elements. (We will take $k \geq 2$ to avoid considering complete graphs $J(n, 1)=K_{n}$, while the isomorphism $J(n, k) \cong J(n, n-k)$ given by taking complements allows us to assume that $k \leq n / 2$.) As observed recently by Dobson and Malnič [11], Godsil's result extends to the Johnson graphs $J(n, k)$ with $2 \leq k \leq(n-1) / 2$, since they also have automorphism groups isomorphic to $S_{n}$. The same authors, as a corollary to a study of groups acting on partitions, have obtained similar results for the Johnson graphs $J(n, n / 2)$ and for the folded Johnson graphs, quotients of $J(n, n / 2)$ by complementation.

The distance $i$ Johnson graphs $J(n, k)_{i}$, for $i=1, \ldots, k$, have vertex set $\left(\begin{array}{l}N \\ k\end{array}\right)$, but with pairs of vertices adjacent if and only if they are at distance $i$ in $J(n, k)$, that is, if and only if the corresponding subsets of $N$ have $k-i$ elements in common. Thus $J(n, k)=J(n, k)_{1}$ and $K(n, k)=J(n, k)_{k}$, for example. If $I$ is any non-empty subset of $\{1, \ldots, k\}$ one can form the merged Johnson graph

$$
J(n, k)_{I}=\bigcup_{i \in I} J(n, k)_{i},
$$

in which two $k$-element subsets of $N$ are adjacent if and only if they are at distance $i$ in $J(n, k)$ for some $i \in I$.

The automorphism groups of the merged Johnson graphs $J=J(n, k)_{I}$ have been described in [17, Theorem 2], repeated here as Theorem 5.1. For most values of the parameters, Aut $J=S_{n}$. However, in some cases, Aut $J$ is significantly larger than $S_{n}$ and contains $S_{n}$ as a proper subgroup. This makes it impossible to extend the results of Godsil or of Dobson and Malnič mentioned above along the same lines to such graphs. In the following theorem we use different techniques to extend their results to all the merged Johnson graphs $J(n, k)_{I}$. Perhaps surprisingly, none of the cases where Aut $J$ contains $S_{n}$ as a proper subgroup yields new Cayley graphs, apart from some rather trivial examples in cases (4) and (5), where the automorphism group is particularly large.

Theorem 1.1. Let $2 \leq k \leq n / 2$ and let $I$ be a non-empty subset of $\{1, \ldots, k\}$. Then the merged Johnson graph $J=J(n, k)_{I}$ has a regular group $G$ of automorphisms if and only if one of the following holds:

(1) $n$ is a prime power, $n \equiv 3 \bmod (4)$ and $k=2$, with any $I$, and $G \cong A H L_{1}(F)$ acting on some Dickson near-field $N=F$ of order $n$;

(2) $n=8$ and $k=3$, with any $I$, and $G \cong A G L_{1}(8)$ acting on the finite field $N=\mathbb{F}_{8}$;

(3) $n=32$ and $k=3$, with any $I$, and $G \cong A \Gamma L_{1}(32)$ acting on the finite field $N=\mathbb{F}_{32}$;

(4) $I=\{1, \ldots, k\}$, with any $n$ and $k$, and $G$ any group of order $\left(\begin{array}{l}n \\ k\end{array}\right)$, acting on itself by right multiplication; 
(5) $k=n / 2$ and $I=\{k\}$ or $\{1, \ldots, k-1\}$, with $G$ any group of order $\left(\begin{array}{l}n \\ k\end{array}\right)$, acting on itself by right multiplication.

This result characterises those graphs $J(n, k)_{I}$ which are Cayley graphs, if we exclude those with $k=n / 2$ and $I=\{k\}$, which are not connected.

Here, if $F$ is a Dickson near-field then $A \Gamma L_{1}(F)$ is the group of transformations $t \mapsto t^{\gamma} a+b$ of $F$, where $a, b \in F, a \neq 0$ and $\gamma$ is an automorphism of $F$, while $A G L_{1}(F)$ is the subgroup for which $\gamma$ is the identity. If $n$ is odd then we denote by $A H L_{1}(F)$ the subgroup of index 2 in $A G L_{1}(F)$ for which $a$ is a non-zero square (here ' $H$ ' stands for 'half'). Each field is a near-field, and when $F$ is the finite field $\mathbb{F}_{n}$ of order $n$ we write simply $A \Gamma L_{1}(n)$, etc. (See [6, §1.12], [10, §7.6], [14, §20.7] or the Appendix for near-fields and their associated groups.)

The actions in Theorem 1.1(1) for which $F$ is a field can be explained and constructed using maps on surfaces. The graph $J(n, 2)$ is isomorphic to the line graph $L\left(K_{n}\right)$ of the complete graph $K_{n}$. A construction due to Biggs [2] shows that for each prime power $n \equiv 3 \bmod (4)$, $A H L_{1}(n)$ acts regularly on the edges of an orientably regular map with 1-skeleton $K_{n}$, inducing a regular group of automorphisms of $J(n, 2)$. There are similar interpretations, in terms of maps on surfaces, for the actions in parts (2) and (3) of Theorem 1.1.

Theorem 1.1 is proved in Section 7, using the description of Aut $J$ in Theorem 5.1, The cases where Aut $J=S_{n}$ are dealt with as in Godsil's theorem, by using known results on $k$-homogeneous groups. This method also shows that if $5<k<(n-1) / 2$, and in addition in most cases where $k=4,5$ or $(n-1) / 2$, the groups $A_{n}$ and $S_{n}$ are the only vertex-transitive groups of automorphisms of $J$, again extending a result of Godsil [13] for the odd graphs $O_{k+1}=J(2 k+1, k)_{k}$; see Corollary 5.2 for details. However, those cases where Aut $J>S_{n}$ require other methods, based in some cases, for example, on considering $G \cap S_{n}$ as a permutation group on $N$.

Theorem 1.1 was motivated by a paper of Gauyacq [12, in which the author studies quasiCayley graphs. These are graphs with a regular family $\mathcal{F}$ of automorphisms, meaning that for each ordered pair $(v, w)$ of vertices there is a unique automorphism in $\mathcal{F}$ taking $v$ to $w$. This generalises the concept of a Cayley graph, where $\mathcal{F}$ is a group of automorphisms. In [15] we generalise this further to the concept of an $r$-regular family of automorphisms, where there are exactly $r$ elements of $\mathcal{F}$ taking $v$ to $w$. The following theorem characterises those merged Johnson graphs which have a 2-regular group of automorphisms (by which we mean one which is transitive, with vertex-stabilisers of order 2). For instance, this class of graphs includes the well-known example of the Petersen graph $O_{3}=J(5,2)_{2}$ in Theorem $1.2(1)$, where we take $F=\mathbb{F}_{5}$.

Theorem 1.2. Let $2 \leq k \leq n / 2$ and let $I$ be a non-empty subset of $\{1, \ldots, k\}$. Then $G$ is a 2-regular group of automorphisms of the graph $J=J(n, k)_{I}$ if and only if one of the following holds: 
(1) $n$ is a prime power, $k=2$, with any $I$, and $G \cong A G L_{1}(F)$ for some near-field $N=F$ of order $n$

(2) $n=6$ and $k=3$, where $G \cong A G L_{1}(5) \times S_{2}$, with any $I$, and $A G L_{1}(5)$ acting naturally on the projective line $N=\mathbb{P}^{1}\left(\mathbb{F}_{5}\right)$, fixing $\infty$, and $S_{2}$ generated by complementation in $N$;

(3) $n=10, k=5$ and $I=\{1,4\},\{2,3\},\{1,4,5\}$ or $\{2,3,5\}$, where $G \cong P S L_{2}(8)$ (this action of $G$ is explained in Section 8 );

(4) $n$ is even, $k=n / 2$ and $I=\{k\}$ or $\{1, \ldots, k-1\}$, where $G$ is any group of order $2\left(\begin{array}{l}n \\ k\end{array}\right)$ with a non-normal subgroup $H$ of order 2 , acting on the cosets of $H$;

(5) $I=\{1, \ldots, k\}$, where $G$ is any group of order $2\left(\begin{array}{l}n \\ k\end{array}\right)$ with a non-normal subgroup $H$ of order 2 , acting on the cosets of $H$.

The structure of the proof is similar to that for Theorem 1.1, though the details are rather different. In the cases where Aut $J=S_{n}$, for instance, we need to consider groups which are $k$-homogeneous with stabilisers of $k$-element subsets having order 2 , rather than 1 . Proving the existence and essential uniqueness of the 2-regular action of $P S L_{2}(8)$ in Theorem $1.2(3)$ requires non-trivial results from the cohomology of groups, such as Shapiro's Lemma; we are grateful to Ian Leary for suggesting the use of this result.

Sabidussi [24] and Godsil [13], using different but equivalent definitions, have introduced a parameter $d(\Gamma)$, called deviation or deficiency, which measures the degree to which a vertex-transitive graph $\Gamma$ differs from being a Cayley graph. We will call this the Cayley deficiency of $\Gamma$, and will use Godsil's definition of $d(\Gamma)$ as the least order of the vertexstabilisers in any vertex-transitive group of automorphisms of $\Gamma$ (see Section 6). Thus $d(\Gamma)=1$ if and only if $\Gamma$ is a Cayley graph, and one can view Theorems 1.1 and 1.2 as classifying those merged Johnson graphs $J=J(n, k)_{I}$ which have Cayley deficiency $d(J)=1$ or 2 . By contrast, for most choices of $n, k$ and $I$, the Cayley deficiency $d(J)$ increases faster than exponentially as $n \rightarrow \infty$, so that $J$ is very far from being a Cayley graph.

\section{JOHNSON GRAPHS AND THEIR AUTOMORPHISMS}

The Johnson graphs $J(n, k)$, the distance $i$ Johnson graphs $J(n, k)_{i}$, the Kneser graphs $K(n, k)=J(n, k)_{k}$ and the merged Johnson graphs $J(n, k)_{I}$, defined in Section 1, all have as their vertex-set the set $\left(\begin{array}{l}N \\ k\end{array}\right)$ of $k$-element subsets of an $n$-element set $N$; we will always assume that $2 \leq k \leq n / 2$, and that $\emptyset \neq I \subseteq\{1, \ldots, k\}$. We will consider all of these graphs as particular cases of the graph $J:=J(n, k)_{I}$, where two $k$-element subsets $K, K^{\prime} \subseteq N$ are adjacent in $J$ if and only if $\left|K \cap K^{\prime}\right|=k-i$ for some $i \in I$. We will generally take $N$ to be the set $\{1, \ldots, n\}$, though in some cases we may identify it with a structure such as a field or projective line with $n$ elements.

The symmetric group $S_{n}$, acting naturally on $N$, preserves the cardinalities of intersections of subsets of $N$, so it is embedded as a subgroup of Aut $J$ for each $J$. In most cases $S_{n}$ is the full automorphism group of $J$ : the exceptions are listed later in Theorem 5.1. In 
particular, Aut $J=S_{n}$ in all cases where $2 \leq k<(n-1) / 2$, with the exception of the complementary graphs $J(12,4)_{I}$ for $I=\{1,3\}$ and $\{2,4\}$. For this reason, we will first study groups $G$ of automorphisms of graphs $J=J(n, k)_{I}$ arising from subgroups of $S_{n}$.

\section{3. $k$-HOMOGENEOUS GROUPS}

A subgroup $G$ of $S_{n}$ is vertex-transitive on the graph $J=J(n, k)_{I}$ if and only if it acts transitively on $\left(\begin{array}{l}N \\ k\end{array}\right)$, that is, $G$ is a $k$-homogeneous permutation group. Such groups have been classified, and we list them for each $k \geq 2$ later in this section. (We omit the case $k=1$, since 1-homogeneity is equivalent to transitivity, and $J(n, 1)_{1}$ is isomorphic to the complement of $K_{n}$.)

First of all, it is clear that any $k$-transitive group is $k$-homogeneous. For each $k \geq 2$ the $k$-transitive finite groups are known as a result of the classification of finite simple groups. Apart from the alternating and symmetric groups $A_{n}$ and $S_{n}$, the multiply transitive finite groups $G$ form six infinite families and one finite family, consisting of the following:

- affine groups, that is, various subgroups $G \leq A \Gamma L_{d}(q)$, of degree $n=q^{d}$ for some prime power $q$ and $d \geq 1$;

- projective groups, of degree $n=\left(q^{d}-1\right) /(q-1)$ for some prime power $q$ and $d \geq 2$, such that $P S L_{d}(q) \leq G \leq P \Gamma L_{d}(q)$;

- unitary groups, of degree $n=q^{3}+1$ for some prime power $q$, such that $\operatorname{PSU}_{3}(q) \leq$ $G \leq P \Gamma U_{3}(q)$

- symplectic groups $G=S p_{2 d}(2)$, each with two representations of degrees $n=$ $2^{d-1}\left(2^{d} \pm 1\right)$, for some $d \geq 3$;

- Suzuki groups, of degree $n=q^{2}+1$ for some $q=2^{e}$ with odd $e \geq 3$, such that $S z(q) \leq G \leq$ Aut $S z(q)$

- Ree groups, of degree $n=q^{3}+1$ for some $q=3^{e}$ with odd $e \geq 3$, such that $\operatorname{Re}(q) \leq G \leq \operatorname{Aut} \operatorname{Re}(q)$

- a finite number of sporadic examples: the Mathieu groups $M_{n}$ for $n=11,12,22,23$ and 24, and Aut $M_{22}$ for $n=22$, acting on Steiner systems; $P S L_{2}(11)$ acting on the cosets of $A_{5}$ for $n=11 ; M_{11}$ on the cosets of $P S L_{2}(11)$ for $n=12 ; A_{7}$ on the cosets of $A G L_{3}(2)$ for $n=15 ; P \Sigma L_{2}(8) \cong R e(3)$ on its Sylow 3 -subgroups for $n=28$; the Higman-Sims group $H S$ for $n=176$; the Conway group $\mathrm{Co}_{3}$ for $n=276$.

(Apart from the affine groups, the groups listed here are all finite simple or almost simple groups; see [8] or [25] for the definitions and general properties of these groups. For a detailed description of them as multiply transitive permutation groups, see [10, §7.7], though it omits Aut $M_{22}$, or [6. Chapter 7] for a more concise but complete list.) The degrees of the groups in these seven families form a subset of $\mathbb{N}$ of asymptotic density 0 ; see [7] for a more precise estimate of the density of this set.

None of the groups in this list is 6-transitive, and the only 5-transitive groups are $M_{12}$ and $M_{24}$. In addition to these, the only 4-transitive groups are $M_{11}$ and $M_{23}$, and the only 
3-transitive groups are various subgroups of $A G L_{d}(2)$ and $P \Gamma L_{2}(q)$, together with $M_{11}$ (for $n=12), M_{22}$ and Aut $M_{22}$. All the remaining groups are merely 2-transitive.

In most cases the converse is also true, that is, a $k$-homogenous group is in fact $k$-transitive. The following theorem, based on results of Livingstone and Wagner [20] and Kantor [19], shows where the exceptions occur:

Theorem 3.1. Let $G$ be a $k$-homogeneous permutation group of finite degree $n$, where $2 \leq k \leq n / 2$. Then $G$ is $(k-1)$-transitive. Moreover, $G$ is $k$-transitive unless either:

(1) $k=2$ and $G \leq A \Gamma L_{1}(q)$ for some prime power $n=q \equiv 3 \bmod (4)$, or

(2) $k=3$ and $P S L_{2}(q) \leq G \leq P \Gamma L_{2}(q)$ for some prime power $q \equiv 3 \bmod (4)$, with $n=q+1$, or

(3) $k=3$ and $G=A G L_{1}(8), A \Gamma L_{1}(8)$ or $A \Gamma L_{1}(32)$ with $n=8,8$ or 32 , or

(4) $k=4$ and $G=P S L_{2}(8), P \Gamma L_{2}(8)$ or $P \Gamma L_{2}(32)$ with $n=9,9$ or 33.

In each case, the permutation representation of $G$ is the natural one.

(Note that each of the groups $G$ in cases (3) and (4) is $k$-homogeneous but not $k$-transitive, whereas this applies to only some of the groups described in cases (1) and (2): for instance $A \Gamma L_{1}(q)$ and $P \Gamma L_{2}(q)$ are $k$-transitive for $k=2$ and 3 respectively.)

It will be useful to consider, from among the multiply homogeneous groups listed in this section, the set $\mathcal{H}_{k}$ of $k$-homogeneous groups, other than $S_{n}$ and $A_{n}$, for each $k \geq 2$, together with the set $\mathcal{D}_{k}$ of their degrees. Since $\mathcal{H}_{k+1} \subseteq \mathcal{H}_{k}$ for each $k$, it is sufficient to describe the sets $\mathcal{H}_{k} \backslash \mathcal{H}_{k+1}$. We have

- $\mathcal{H}_{k}=\emptyset$ for all $k \geq 6$;

- $\mathcal{H}_{5}=\left\{M_{12}, M_{24}\right\}$

- $\mathcal{H}_{4} \backslash \mathcal{H}_{5}=\left\{M_{11}, M_{23}, P S L_{2}(8), P \Gamma L_{2}(8), P \Gamma L_{2}(32)\right\}$;

- $\mathcal{H}_{3} \backslash \mathcal{H}_{4}$ consists of various subgroups of $A \Gamma L_{d}(2)$ and $P \Gamma L_{2}(q)$, and also $M_{11}$ (for $n=12), M_{22}$, Aut $M_{22}, A G L_{1}(8), A \Gamma L_{1}(8)$ and $A \Gamma L_{1}(32)$;

- $\mathcal{H}_{2} \backslash \mathcal{H}_{3}$ consists of the remaining groups listed above.

By considering the degrees of these groups, stated above, we see that

- $\mathcal{D}_{k}=\emptyset$ for all $k \geq 6$;

- $\mathcal{D}_{5}=\{12,24\}$;

- $\mathcal{D}_{4}=\{9,11,12,23,24,33\}$;

- $\mathcal{D}_{3}$ consists of the integers $2^{d}$ where $d \geq 3$, and $q+1$ where $q$ is a prime power, together with 11,22 and 23;

- $\mathcal{D}_{2}$ consists of all the degrees $n$ given in the list of multiply transitive groups at the start of this section (the groups in Theorem 3.1 contribute no further degrees).

This shows that if $k \geq 6$, or if $2 \leq k \leq 5$ and $n$ avoids an easily described subset of $\mathbb{N}$ of asymptotic density 0 , then the only $k$-homogeneous groups of degree $n$ are $S_{n}$ and $A_{n}$. In fact, $k$-homogeneous groups for $k \geq 2$ are primitive, and Cameron, Neumann and 
Teague [7] have shown that for all but a set of integers $n$ of asymptotic density 0 the only primitive groups of degree $n$ are $S_{n}$ and $A_{n}$.

\section{4. $r$-REGULAR GROUPS FROM $k$-HOMOGENEOUS GROUPS}

Motivated by [12] and [15], we can now apply the information in the preceding section to obtain $r$-regular groups of automorphisms of various merged Johnson graphs from $k$ homogeneous subgroups of $S_{n}$. If $G$ is any vertex-transitive group of automorphisms of $J=J(n, k)_{I}$, it forms an $r$-regular family for $J$ where $r$ is the order $|G| /\left(\begin{array}{l}n \\ k\end{array}\right)$ of the vertexstabilisers. In particular, if $G$ is a $k$-homogeneous subgroup of $S_{n}$ then it acts on $J$ as an $r$-regular group of automorphisms, where $r$ is the order of the subgroup of $G$ stabilising a $k$-element subset $K \subseteq N$.

For us, concerned with Cayley graphs, the case $r=1$ is the most important. The corresponding subgroups $G$ of $S_{n}$ are the sharply $k$-homogeneous groups, those acting regularly on $k$-element subsets, so if such a group $G$ exists then $J(n, k)_{I}$ is a Cayley graph. Since $k \geq 2, G$ cannot be $k$-transitive, so it must be one of the exceptional groups described in Theorem 3.1. Case (1), where $k=2$, has been dealt with by Kantor in [18. Case (2), with $k=3$, cannot arise, since $G$ contains the element $t \mapsto 1 /(1-t)$ of $P S L_{2}(q)$, which preserves the subset $\{0,1, \infty\}$. Cases (3) and (4) are easily dealt with by comparing $|G|$ with $\left(\begin{array}{l}n \\ k\end{array}\right)$. As a result, we have the following well-known easy consequence of [19, Theorem 1]:

Corollary 4.1. Let $G$ be a permutation group $G$ of finite degree $n$. Then $G$ is sharply $k$-homogeneous for some $k \geq 2$ if and only if either

(1) $k=2$ and $G \cong A H L_{1}(F)$ for some Dickson near-field $F$ of prime power order $n \equiv 3 \bmod (4)$, or

(2) $k=3$ and $G \cong A G L_{1}(8)$ or $A \Gamma L_{1}(32)$.

It follows that $J(n, k)_{I}$ is a Cayley graph in each of these cases, for any non-empty set $I$.

The case $r=2$ is also of interest. For later use, in proving Theorem 1.2 and in [15], we also note that if $F$ is a near-field of (necessarily prime power) order $n$ then the group $G=A G L_{1}(F)$ is sharply 2-transitive on $F$, so it acts 2-regularly on $J(n, 2)_{I}$ for any $I$.

\section{Automorphism Groups of MERGED Johnson GRAPHS}

The above examples all arise from subgroups of $S_{n}$, which has an induced action on each merged Johnson graph $J=J(n, k)_{I}$. However, the following result [17, Theorem 2] shows that in some cases Aut $J$ is strictly larger than $S_{n}$, leading to the possibility of further vertex-transitive groups of automorphisms. First we need some notation: define $e=\left(\begin{array}{l}n \\ k\end{array}\right) / 2$, and for any $I \subseteq\{1, \ldots, k\}$ define $I^{\prime}=I \backslash\{k\}$ and $I^{\prime \prime}=k-I^{\prime}=\left\{k-i \mid i \in I^{\prime}\right\}$. We will assume that $I \neq\{1, \ldots, k\}$, since otherwise $J(n, k)_{I}$ is a complete graph and Aut $J$ is the symmetric group on $\left(\begin{array}{l}N \\ k\end{array}\right)$. 
Theorem 5.1. [17, Theorem 2] Let $J=J(n, k)_{I}$ where $2 \leq k \leq n / 2$ and $I$ is a non-empty proper subset of $\{1, \ldots, k\}$.

(1) If $2 \leq k<(n-1) / 2$, and $J \neq J(12,4)_{I}$ with $I=\{1,3\}$ or $\{2,4\}$, then Aut $J=S_{n}$.

(2) If $(n, k)=(12,4)$ with $I=\{1,3\}$ or $\{2,4\}$, then Aut $J=G O_{10}^{-}(2)$.

(3) If $k=(n-1) / 2$ and $I \neq k+1-I$, then Aut $J=S_{n}$.

(4) If $k=(n-1) / 2$ and $I=k+1-I$, then Aut $J=S_{n+1}$.

(5) If $k=n / 2$ and $I \neq\{k\}$ or $\{1, \ldots, k-1\}$, and $I^{\prime} \neq I^{\prime \prime}$, then Aut $J=S_{2} \times S_{n}$.

(6) If $k=n / 2$ and $I \neq\{k\}$ or $\{1, \ldots, k-1\}$, and $I^{\prime}=I^{\prime \prime}$, then Aut $J=S_{2}^{e} \rtimes S_{n}$.

(7) If $k=n / 2$ and $I=\{k\}$ or $\{1, \ldots, k-1\}$, then Aut $J=S_{2}^{e} \rtimes S_{e}=S_{2} \succ S_{e}$.

In all cases, Aut $J$ contains $S_{n}$ with its induced action on $k$-element subsets. In cases (1) and (3) this is the full automorphism group, whereas in the remaining cases, namely (2) and (4) to (7), the automorphism groups are larger; these are described in Section 7, in the proof of Theorem 1.1, where we first need to use them.

Corollary 5.2. Let $J=J(n, k)_{I}$ where $I$ is a non-empty proper subset of $\{1, \ldots, k\}$. Suppose that either

(1) $5<k<(n-1) / 2$, or

(2) $5<k=(n-1) / 2$ and $I \neq k+1-I$, or

(3) $5=k<(n-1) / 2$ and $n \neq 12,24$, or

(4) $4=k<(n-1) / 2$ and $n \neq 9,11,12,23,24,33$.

Then the only vertex-transitive groups of automorphisms of $J$ are $A_{n}$ and $S_{n}$, each acting naturally.

Proof. Theorem 5.1] ensures that Aut $J=S_{n}$ in all these cases, so that any vertex-transitive group of automorphisms of $J$ must be a $k$-homogeneous subgroup of $S_{n}$. As shown in Section 3, for $k>5$ the only such groups are $A_{n}$ and $S_{n}$. The same conclusion applies for $k=5$ provided $n \neq 12,24$, so that the 5 -homogeneous Mathieu groups of those degrees are avoided. Similarly, one can take $k=4$ provided $n \neq 9,11,12,23,24,33$, so that case (2) of Theorem 5.1 and the 4 -homogeneous groups in $\mathcal{H}_{4}$ are avoided.

Similar results apply for $k=2$ and $k=3$, but in each case one has to exclude an infinite set $\mathcal{H}_{k}$ of values of $n$, of asymptotic density 0 .

\section{Deviation And DEFICIEnCY}

As mentioned in the Introduction, Cayley graphs are believed to constitute a significant part of the class of vertex-transitive graphs. By definition, every vertex-transitive graph admits a vertex-transitive group of automorphisms, which may or may not be its full automorphism group. By a well-known result of Sabidussi [24], each Cayley graph $\Gamma$ admits a vertex-transitive group of automorphisms $G$ of order $|G|=|V(\Gamma)|$, which again may or may not be the full automorphism group. Thus the ratio between the order of 
a smallest vertex-transitive subgroup of Aut $\Gamma$ (rather than that of Aut $\Gamma$ itself) and the order of the graph $\Gamma$ appears to be a good indicator of how far $\Gamma$ is from being a Cayley graph.

Other such indicators have also been proposed. In [24] Sabidussi defined the deviation $\operatorname{dev}(\Gamma)$ of a vertex-transitive graph $\Gamma$ to be the smallest integer $r$ such that the lexicographic product $\Gamma\left[\overline{K_{r}}\right]$ is a Cayley graph. (Here $\bar{K}_{r}$ is the null graph with $r$ vertices and no edges, and the lexicographic product $\Gamma\left[\overline{K_{r}}\right]$ is a covering of $\Gamma$ consisting of $|V(\Gamma)|$ disjoint copies of $\overline{K_{r}}$, each forming the fibre over a vertex of $\Gamma$, with two such fibres joined to make a complete bipartite graph $K_{r, r}$ if and only if the vertices they cover are adjacent in $\Gamma$.) In particular $\Gamma\left[\overline{K_{1}}\right] \cong \Gamma$, so $\operatorname{dev}(\Gamma)=1$ if and only if $\Gamma$ is a Cayley graph.

Godsil [13] defined the deficiency $d(\Gamma)$ of a vertex-transitive graph $\Gamma$ to be the least order $\left|G_{v}\right|$ of the vertex-stabilisers $G_{v}(v \in V(\Gamma))$ in any vertex-transitive group $G$ of automorphisms of $\Gamma$. Again, $d(\Gamma)=1$ if and only if $\Gamma$ is a Cayley graph.

In [16], Malnič, Marušič and the second author showed that if $G$ is any vertex-transitive group of automorphisms of a graph $\Gamma$, then the Cayley graph of $G$, with the connecting set consisting of the elements of $G$ mapping a specific vertex $v$ of $\Gamma$ to its neighbors, is isomorphic to $\Gamma\left(\overline{K_{r}}\right)$ where $r=\left|G_{v}\right|$. Thus Sabidussi's deviation $\operatorname{dev}(\Gamma)$ is equal to Godsil's deficiency $d(\Gamma)$, and since $\left|G_{v}\right|=|G| /|V(\Gamma)|$, it is also equal to the ratio of the order of a smallest vertex-transitive group of automorphisms and the order of the graph — the parameter we discussed at the beginning of this section. Thus all three are actually the same parameter, measuring how much a vertex-transitive graph deviates from being a Cayley graph. In this paper, we will call this parameter the Cayley deficiency $d(\Gamma)$. The bigger the Cayley deficiency, the further the graph is from being a Cayley graph, with Cayley graphs being those of Cayley deficiency 1.

For example, if $\Gamma$ is one of the merged Johnson graphs $J=J(n, k)_{I}$ listed in Corollary 5.2 , the smallest vertex-transitive subgroup of Aut $J$ is $A_{n}$, so $d(J)=n ! / 2\left(\begin{array}{l}n \\ k\end{array}\right)=k !(n-k) ! / 2$. If $n \rightarrow \infty$ with $k$ fixed, then $d(J)$ grows faster than exponentially, while the order $\left(\begin{array}{l}n \\ k\end{array}\right)$ of $J$ has polynomial growth, of degree $k$. Thus if $n \gg k>5$ then $J$ is very far from being a Cayley graph.

On the other hand, even though the automorphism group of a merged Johnson graph $J(n, k)_{I}$ always contains $S_{n}$, in cases other than those listed in Corollary 5.2 the group $A_{n}$ need not be a smallest vertex-transitive subgroup of Aut $J$. For instance the $r$-regular groups of automorphisms arising from $k$-homogeneous permutation groups, discussed in Section 4, give examples of this with relatively small values of $r$, and hence of $d(J)$ since $d(J) \leq r$. The aim of the rest of this paper is to classify the merged Johnson graphs $J$ with the smallest Cayley deficiencies, namely those with $d(J)=1$ or 2 . 


\section{Cayley properties of merged Johnson Graphs}

We are now ready to prove Theorem 1.1, which classifies the merged Johnson graphs of Cayley deficiency $d(J)=1$.

Proof. We first deal with two trivial cases. If $I=\{1, \ldots, k\}$, as in Theorem 1.1(4), then the graph $J:=J(n, k)_{I}$ is a complete graph on $\left(\begin{array}{l}n \\ k\end{array}\right)$ vertices; the groups $G$ which can then act regularly as a group of automorphisms of $J$ are those of order $\left(\begin{array}{l}n \\ k\end{array}\right)$, so that $J$ is a Cayley graph for $G$ with respect to the generating set $G \backslash\{1\}$.

Similarly, if $k=n / 2$ and $I=\{1, \ldots, k-1\}$, as in conclusion (5), then $J$ is this same complete graph minus a complete matching, given by complementation; in this case $G$ can act as a regular group of automorphisms of $J$ if and only if it has order $\left(\begin{array}{l}n \\ k\end{array}\right)$, so that $J$ is a Cayley graph for $G$ with respect to the generating set $G \backslash T$ for some subgroup $T$ of order 2 (which exists since $\left(\begin{array}{l}n \\ k\end{array}\right)$ is even). The same regular groups arise if $k=n / 2$ and $I=\{k\}$, giving the complementary graph $J$, the disjoint union of $e=\left(\begin{array}{l}n \\ k\end{array}\right) / 2$ copies of $K_{2}$; however, this graph is not connected, so it is not a Cayley graph. Having dealt with these cases, we will assume for the rest of this proof that $I \neq\{1, \ldots, k\}$, and that if $k=n / 2$ then $I$ is neither $\{k\}$ nor $\{1, \ldots, k-1\}$.

In each of cases (1), (2) and (3), Corollary 4.1 shows that the specified group $G$ acts sharply $k$-homogeneously on an $n$-element set $N$, inducing a regular group of automorphisms of $J$.

It remains to prove the converse, that these are the only cases in which $J$ is a Cayley graph for a group $G$. We will do this by a case-by-case analysis of the various possibilities for Aut $J$ (and hence for $G$ ), as classified in Theorem 5.1.

(a) First suppose that $G$ is contained in $S_{n}$, the symmetric group on $N$. (By Theorem 5.1 this includes all cases in which $k<(n-1) / 2$ except $J=J(12,4)_{I}$ with $I=\{1,3\}$ or $\{2,4\}$.) Then $G$ acts on $N$ as a sharply $k$-homogeneous group, so Corollary 4.1 implies that $G$ and $J$ satisfy conclusion (1), (2) or (3). (This is essentially the argument used by Godsil [13] to deal with the Kneser graphs $K(n, k)$; as noted by Dobson and Malnič [11] it also applies to the Johnson graphs $J(n, k)$ with $k<n / 2$.)

(b) Next we deal with the exceptional graphs $J=J(12,4)_{I}$, with $I=\{1,3\}$ or $\{2,4\}$. By Theorem 5.1 these two complementary graphs have as their automorphism group the general orthogonal group $\mathrm{GO}_{10}^{-}(2)$ in ATLAS notation [8], so it is sufficient to show that this group has no subgroup $G$ of order $\left(\begin{array}{c}12 \\ 4\end{array}\right)=495=3^{2} .5 .11$. Having odd order, any such group must be contained in the simple subgroup $S=O_{10}^{-}(2)$ of index 2 in $G O_{10}^{-}(2)$.

If $n_{p}$ denotes the number of Sylow $p$-subgroups of $G$, then Sylow's theorems give $n_{3}=1$ or $55, n_{5}=1$ or 11 , and $n_{11}=1$ or 45 . If $n_{5}=1$ then $G$ has a normal Sylow 5 -subgroup $K \cong C_{5}$; since $\mid$ Aut $K \mid=4$, elements of order 11 must centralise $K$, whereas the ATLAS shows that $S$ has no elements of order 55. Thus $n_{5}=11$. 
If $n_{11}=45$ there are $45.10=450$ elements of order 11 in $G$; since there are also $11.4=44$ elements of order 5 , and one of order 1 , there are no elements of order 3, contradicting Cauchy's Theorem. Hence $n_{11}=1$, so $G$ has a normal Sylow 11-subgroup $E \cong C_{11}$.

A Sylow 3-subgroup $T$ of $G$ has order 9, coprime to $\mid$ Aut $E \mid=10$, so it centralises $E$. However, according to the ATLAS, $\left|C_{S}(g)\right|=33$ for each element $g \in S$ of order 11, a contradiction. Thus Aut $J$ contains no subgroup $G$ of order 495, so $J$ is not a Cayley graph.

Parts (a) and (b) deal with all cases where $k<(n-1) / 2$. To make further progress we need a technical lemma. In order to maintain continuity we postpone its proof to the end of this section.

Lemma 7.1. Let $n \geq 4$ and $k=n / 2$. Then a subgroup $H \leq S_{n}$ has two orbits on $k$-element subsets of $N=\{1, \ldots, n\}$, each of them regular, if and only if $n=4$ and $H \cong C_{3}$.

(c) Now suppose that $n$ is even and $k=n / 2$. We have already dealt with the cases $I=\{1, \ldots, k\},\{1, \ldots, k-1\}$ and $\{k\}$, so we may assume that $n>4$. If $n>4$ and $k=n / 2$, Theorem 5.1 shows that Aut $J$ is a direct or semidirect product $S_{n} \times S_{2}$ or $S_{2}^{e} \rtimes S_{n}$ as $I^{\prime} \neq I^{\prime \prime}$ or $I^{\prime}=I^{\prime \prime}$ (recall that $e=\left(\begin{array}{l}n \\ k\end{array}\right) / 2, I^{\prime}=I \backslash\{k\}$ and $I^{\prime \prime}=k-I^{\prime}$ ).

First, suppose that $I^{\prime} \neq I^{\prime \prime}$, so that Aut $J=S_{n} \times S_{2}$, with $S_{n}$ acting naturally and $S_{2}$ generated by the automorphism sending each $k$-element subset to its complement. Part (a) of this proof shows that there are no regular subgroups $G \leq S_{n}$, so any regular subgroup $G$ must have a subgroup $H=G \cap S_{n}$ of index 2, with two regular orbits on $k$-element subsets of $N$. Lemma 7.1 then shows that $n=4$, against our assumptions, so this possibility is eliminated.

Now suppose that $I^{\prime}=I^{\prime \prime}$, so that Aut $J=S_{2}^{e} \rtimes S_{n}$; in this case the complement $S_{n}$ in this semidirect product acts naturally, while each direct factor $S_{2}$ of the normal subgroup $S_{2}^{e}$ is generated by an involution transposing one $k$-element subset with its complement, and leaving all others invariant. It follows that the only elements of $S_{2}^{e}$ acting on $J$ without fixed points are those in the diagonal subgroup, transposing every $k$-element subset with its complement. Thus $\left|G \cap S_{2}^{e}\right| \leq 2$, and we can apply the argument of the preceding paragraph to the image of $G$ in $S_{n}$, with the same conclusion.

(d) Finally, let $n$ be odd and $k=(n-1) / 2$, so that Aut $J=S_{n}$ or $S_{n+1}$ where $I \neq k+1-I$ or $I=k+1-I$ respectively. In the first case part (a) of this proof applies, so we may assume that $I=k+1-I$ and Aut $J=S_{n+1}$.

In this case, $S_{n+1}$ acts on $J$ as follows. Let $N^{*}=N \cup\{n+1\}=\{1, \ldots, n+1\}$, and let $\Phi$ denote the set of all equipartitions of $N^{*}$, meaning the unordered pairs of complementary subsets $P_{1}, P_{2}$ of $N^{*}$ with $\left|P_{i}\right|=(n+1) / 2$ for each $i=1,2$. There is a bijection $\beta:\left(\begin{array}{l}N \\ k\end{array}\right) \rightarrow \Phi$, sending each $K \in\left(\begin{array}{l}N \\ k\end{array}\right)$ to the equipartition $\{K \cup\{n+1\}, N \backslash K\}$; its inverse sends each $\left\{P_{1}, P_{2}\right\} \in \Phi$ to $P_{i} \backslash\{n+1\}$ where $n+1 \in P_{i}$. The natural action of $S_{n+1}$ on $N^{*}$ and hence on $\Phi$ induces, via $\beta$, an action on $\left(\begin{array}{l}N \\ k\end{array}\right)$ preserving $J$; its restriction to the subgroup fixing $n+1$ is the natural action of $S_{n}$ on the vertex set $N=\{1,2, \ldots, n\}$ of $J$. 
We need to show that Aut $J$ has no subgroup $G$ acting regularly on $\left(\begin{array}{l}N \\ k\end{array}\right)$, or equivalently that $S_{n+1}$ has no subgroup $H$ acting regularly on $\Phi$. Such a subgroup would be a group of degree $n+1$ on $N^{*}$ with two regular orbits on $(k+1)$-element subsets, where $k+1=(n+1) / 2$; Lemma 7.1, with a slight change of notation, then implies would imply that $n=3$ and so $k=1$, against our assumption that $k \geq 2$.

Remark. It is straightforward to check that in each of conclusions (2) and (3) of Theorem 1.1 there is a unique conjugacy class of regular subgroups $G$ in Aut $J$, while in (1) the conjugacy classes correspond to the isomorphism classes of Dickson near-fields $F$ of order $n$; see more on this in the Appendix.

Lemma 7.1, used in the above proof, is the particular case $r=1$ of the following lemma, the other cases of which we will need later.

Lemma 7.2. Let $n=2 k \geq 4$. Then a subgroup $H \leq S_{n}$ has two orbits on $k$-element subsets of $N=\{1, \ldots, n\}$, both of them $r$-regular for some $r \leq 4$, if and only if

(1) $r=1, n=4$ and $H \cong A_{3}$, or

(2) $r=2, n=4$ and $H \cong S_{3}$, or

(3) $r=2, n=6$ and $H \cong A G L_{1}(5)$, or

(4) $r=4, n=10$ and $H \cong P S L_{2}(8)$,

with $H$ fixing a point and having its natural transitive action on the remaining $n-1$ points. The two orbits on k-element subsets then consist of those subsets containing or not containing the fixed point.

Proof. Livingstone and Wagner [20] have shown that if $s \leq t$ and $s+t \leq n$, then a group of degree $n$ has at least as many orbits on $t$-element subsets as it has on $s$-element subsets (see also Cameron [5, Theorem 2.2]). It follows that a group $H$ satisfying the hypotheses of this lemma must have at most two orbits on $m$-element subsets, for each $m=1, \ldots, k$.

If $H$ is intransitive on $N$, it has two orbits $N_{1}$ and $N_{2}$, and we may assume that $\left|N_{1}\right| \geq\left|N_{2}\right|$. If $\left|N_{2}\right| \geq 2$ then since $\left|N_{1}\right| \geq k \geq 2, H$ must have at least three orbits on $k$-element subsets, each consisting of such subsets $K$ with $\left|K \cap N_{2}\right|=0,1$ or 2 . This contradicts our hypothesis, so $\left|N_{2}\right|=1$ and the two orbits on $k$-element subsets consist of those $K$ containing or not containing $N_{2}$.

This shows that $H$ is a $(k-1)$-homogeneous group of odd degree $n-1=2(k-1)+1$ on $N_{1}$. It follows that $H$ is set-transitive on $N_{1}$, by which we mean that $H$ is $m$-homogeneous for all $m \leq n-1$. Set-transitive groups have been classified by Beaumont and Peterson [1]. Excluding groups of even degree (alternating and symmetric groups, and $P G L_{2}(5)$ of degree 6 ), the only possibilities for $H$ are the following, with $r$ denoting the size of the stabiliser of a $(k-1)$-element subset of $N_{1}$ :

- $A_{n-1}$ and $S_{n-1}$ for even $n \geq 4$, with $r=(k-1) ! k ! / 2$ and $(k-1) ! k !$;

- $A G L_{1}(5)$ of degree $n-1=5$, with $r=2$; 
- $P S L_{2}(8)$ and $P \Gamma L_{2}(8)$ of degree $n-1=9$, with $r=4$ and 12.

Only $A_{3}, S_{3}, A G L_{1}(5)$ and $P S L_{2}(8)$ satisfy $r \leq 4$, giving conclusions (1) to (4). We may therefore assume that $H$ is transitive on $N$.

Now suppose that $H$ is 2-homogeneous on $N$, or equivalently, since it has even order, 2transitive. (This always applies when $n=4$ or 6 , since $|H|$ is divisible by $\left(\begin{array}{l}n \\ k\end{array}\right) / 2=3$ or 10 .) Inspection of the 2-transitive groups $H$ of even degree $n$ listed in Section 6 shows that they never have two orbits, both of size $\left(\begin{array}{l}n \\ k\end{array}\right) / 2$, on $k$-element subsets for $k=n / 2$ : in most cases, either $|H|$ is not divisible by $\left(\begin{array}{l}n \\ k\end{array}\right) / 2$, or else $H$ preserves some geometric or combinatorial structure on $N$ for which there are at least three non-isomorphic $k$-element subsets.

If $H$ is not 2-homogeneous it has two orbits on pairs, and by taking one of them as the edge set we see that $H$ is a group of automorphisms of a vertex- and edge-transitive graph $\Gamma$ on $N$ of valency $v<k$. Since $n \geq 8$ the following lemma implies that $H$ has at least three orbits on $m$-element subsets for $m=3$ or 4 , giving a contradiction.

In the following lemma, we use the notation $\Gamma_{1}+\Gamma_{2}$ to denote the disjoint union of graphs $\Gamma_{1}$ and $\Gamma_{2}$, and $m \Gamma$ to denote the disjoint union of $m$ copies of a graph $\Gamma$.

Lemma 7.3. Let $\Gamma$ be a regular graph of order $n=2 k \geq 8$ and valency $v$ such that $1 \leq v<k$. Then

(1) if $\Gamma \cong 2 K_{k}$ or $k K_{2}$ there are just two isomorphism classes of induced 3 -vertex subgraphs of $\Gamma$, and three isomorphism classes of induced 4-vertex subgraphs;

(2) otherwise there are at least three isomorphism classes of induced 3-vertex subgraphs.

[Note that there are, up to isomorphism, just four simple graphs on three vertices, namely $K_{3}, K_{2}+K_{1}$ and their complements.]

Proof. If $v=1$ then $\Gamma \cong k K_{2}$ with $k \geq 4$, so the 3 -vertex subgraphs are isomorphic to $K_{2}+K_{1}$ and $3 K_{1}$, and the 4 -vertex subgraphs are isomorphic to $2 K_{2}, K_{2}+2 K_{1}$ and $4 K_{1}$. We may therefore assume that $v \geq 2$.

First suppose that there are no induced subgraphs isomorphic to $P_{3}$, a path of three vertices. Then each vertex $a \in \Gamma$, together with its neighbours, forms a complete graph $K_{v+1}$. It follows that $\Gamma \cong m K_{v+1}$ where $m=n /(v+1) \geq n / k \geq 2$. If $m=2$, so that $\Gamma \cong 2 K_{k}$ with $k \geq 4$, the 3 -vertex subgraphs are isomorphic to $K_{3}$ or $K_{2}+K_{1}$, while the 4 -vertex subgraphs are isomorphic to $K_{4}, K_{3}+K_{1}$ or $2 K_{2}$. If $m \geq 3$ the 3 -vertex subgraphs are isomorphic to $K_{3}, K_{2}+K_{1}$ or $3 K_{1}$ and $\Gamma$ falls under case (2). We may therefore assume that there are induced subgraphs isomorphic to $P_{3}$.

Now suppose that there are no induced subgraphs isomorphic to the null graph $3 K_{1}$. Then for any non-adjacent vertices $a$ and $b$, each remaining vertex is adjacent to $a$ or $b$, so $n \leq 2+2 v=2(v+1) \leq 2 k$. But $n=2 k$, so $\Gamma(a)$ and $\Gamma(b)$ have disjoint vertex-sets, and thus $\Gamma(a) \cup\{a\}$ and $\Gamma(b) \cup\{b\}$ partition $\Gamma$. Each pair of vertices $c, d \in \Gamma(a)$ are adjacent (otherwise $b, c, d$ form an induced subgraph $3 K_{1}$ ), so $\Gamma(a) \cup\{a\} \cong K_{v+1}=K_{k}$. The same 
applies to $b$, so $\Gamma \cong 2 K_{k}$, a case dealt with earlier. We may therefore assume that there are induced subgraphs isomorphic to $3 K_{1}$.

If there are no induced subgraphs isomorphic to $K_{2}+K_{1}$, which has just one edge, then, given any edge $a b$, each vertex $c \neq a, b$ is adjacent to $a$ or $b$, so $n \leq 2+2(v-1)=2 v<2 k$, a contradiction. Thus, apart from the cases $\Gamma \cong 2 K_{k}$ and $k K_{2}$, already dealt with, there are induced subgraphs isomorphic to $P_{3}, K_{2}+K_{1}$ and $3 K_{1}$.

(There are three graphs $\Gamma$ satisfying the hypotheses of Lemma 7.3 when $n=6$ : the graphs $3 K_{2}$ and $2 K_{3}$ each have two isomorphism classes of 3-vertex induced subgraphs, whereas the 6 -cycle $C_{6}$ has three. When $n=4$ the only graph is $2 K_{2}$, with two classes of 2 -vertex subgraphs.

\section{2-REGULAR GROUPS OF AUTOMORPHISMS}

Theorem 1.1 determines those cases where a merged Johnson graph $J$ has a group $G$ of automorphisms acting regularly on the vertices. We will now prove Theorem 1.2, which determines which merged Johnson graphs $J$ have a group of automorphisms acting 2regularly on $J$.

Proof. The structure of the proof follows that of Theorem 1.1, apart from the use of cohomology of groups at one point. We first deal with some trivial cases.

If $I=\{1, \ldots, k\}$ then $J$ is the complete graph on $\left(\begin{array}{l}n \\ k\end{array}\right)$ vertices, so $G$ can be any transitive permutation group with stabilisers of order 2 , or equivalently, any group of order $2\left(\begin{array}{l}n \\ k\end{array}\right)$ acting on the cosets of a core-free (equivalently non-normal) subgroup $H \cong C_{2}$, as in conclusion (5). (Such groups $G$, for example dihedral groups, exist in all cases.) We will therefore assume from now on that $I \neq\{1, \ldots, k\}$.

If $n=2 k$ and $I=\{k\}$ or $\{1, \ldots, k-1\}$ then $J$ is either a complete matching $e K_{2}$ or its complement, where $e=\left(\begin{array}{l}n \\ k\end{array}\right) / 2$, and hence Aut $J=S_{2} \prec S_{e}$, the maximal imprimitive group with $e$ blocks of size 2. In this case the groups $G$ acting 2-regularly are again those of order $2\left(\begin{array}{l}n \\ k\end{array}\right)$ with a non-central subgroup $H \cong C_{2}$, as in conclusion (4): one can identify the vertices with the cosets of $H$ and the blocks with the cosets of a subgroup of order 4 containing $H$ (such subgroups exist since $|G|$ is divisible by 4 ). We will therefore assume from now on that if $n=2 k$ then $I$ is neither $\{k\}$ nor $\{1, \ldots, k-1\}$.

It is straightforward to check that the groups $G$ in conclusions (1) and (2) act 2-regularly on the corresponding graphs $J$, and we will show this for (3) later, under (e). We will now use Theorem 5.1 to prove the converse, that there are no other 2-regular groups of automorphisms than those listed in (1), (2) and (3).

(a) If $G \leq S_{n}$ then $G$ is a $k$-homogeneous permutation group of degree $n$, and the subgroup $G_{K}$ preserving a $k$-element subset $K$ has order 2 .

If $G$ is not $k$-transitive, it is as listed in Theorem 3.1, cases (1) to (4). Having even order, $G$ contains an involution, so if $k=2$ then $G$ acts transitively, not only on unordered pairs 
but also on ordered pairs, that is, it is 2-transitive, against our assumption. Thus $k \geq 3$, so only cases (2), (3) and (4) of Theorem 3.1 apply. Since $\left|G_{K}\right|=2$ we have $|G| /\left(\begin{array}{l}n \\ k\end{array}\right)=2$. However, by inspection of the individual groups, we see that in case (2) this ratio is divisible by 3 , in case (3) it takes the values 1,3 and 1 respectively, and in case (4) it takes the values 4,12 and 4 .

Thus $G$ is $k$-transitive, so $G_{K}$ acts on $K$ as $S_{k}$, and hence $\left|G_{K}\right| \geq k$; however, $\left|G_{K}\right|=2$, so $k=2$, and $J=J(n, 2)_{1}$ or $J(n, 2)_{2}$. Now $G$, as 2-transitive group of degree $n$ and order $n(n-1)$, is sharply 2-transitive. Such groups were classified by Zassenhaus, and are listed in [10, §7.6]: they are the 1-dimensional affine groups $A G L_{1}(F)$ over near-fields $F$ (including the finite fields), and their degrees $n$ are the prime powers, giving conclusion (1).

(b) If $J=J(12,4)_{I}$ with $I=\{1,3\}$ or $\{2,4\}$ then Aut $J \cong G O_{10}^{-}(2)$. Any 2-regular subgroup $G$ has order $2\left(\begin{array}{c}12 \\ 4\end{array}\right)=990$. By the proof of Theorem 1.1 there are no subgroups of order 495 in $G O_{10}^{-}(2)$, so $G$ has no subgroups of index 2 ; it is therefore contained in $O_{10}^{-}(2)$, and hence in one of its maximal subgroups. By [8], the only maximal subgroups of $O_{10}^{-}(2)$ of order divisible by 990 are isomorphic to $A_{12}$ or $C_{3} \times U_{5}(2)$. If $G \leq A_{12}$ then $G$ cannot be transitive on $\{1, \ldots, 12\}$ since $|G|$ is not divisible by 12 ; having order divisible by 11 , it must have orbits of length 11 and 1 , so $G \leq A_{11}$; however, the transitive groups of degree 11 are all known, and there is none of order 990. If $G \leq C_{3} \times U_{5}(2)$, the simple group $U_{5}(2)$ must have a subgroup of order 990 or 330 ; this must be contained in a maximal subgroup of $U_{5}(2)$, whereas by [8] the only maximal subgroups of order divisible by 330 are isomorphic to $P S L_{2}(11)$, a simple group of order 660 with no subgroups of index 2 .

Parts (a) and (b) deal with all cases where $k<(n-1) / 2$. We dealt earlier with the cases where $I=\{1, \ldots, k\}$, and where $k=n / 2$ and $I=\{k\}$ or $\{1, \ldots, k-1\}$, so we may assume that $n>4$.

(c) Now let $k=n / 2$ and $I^{\prime} \neq I^{\prime \prime}$, so that Aut $J=S_{n} \times S_{2}$. Cases where $G \leq S_{n}$ are dealt with in (a), so we may assume that $H:=G \cap S_{n}$ has index 2 in $G$. If $H$ acts transitively on $\left(\begin{array}{l}N \\ k\end{array}\right)$ it does so regularly, so $H$ is $k$-homogeneous but not $k$-transitive on $N$. Theorem 3.1 now implies that $k<n / 2$, against our assumption.

If $H$ is intransitive on $\left(\begin{array}{l}N \\ k\end{array}\right)$ then, having index 2 in a transitive group $G$, it must have two orbits of length $\left(\begin{array}{l}n \\ k\end{array}\right) / 2$, both 2-regular. Since $n>4$, Lemma 7.2 implies that $n=6, k=3$, and $H$ is $A G L_{1}(5)$, acting on $N=\mathbb{P}^{1}(5)$ as the stabiliser of $\infty$ in $P G L_{2}(5)$. Thus $G$ is $A G L_{1}(5) \times C_{2}$, with complementation generating the second factor, as in conclusion (2).

Now suppose that $k=n / 2$ and $I^{\prime}=I^{\prime \prime}$; since $I \neq\{k\},\{1, \ldots, k-1\},\{1, \ldots, k\}$, we must have $n>6$. Then Aut $J=M \rtimes Q$, a semidirect product of a normal subgroup $M \cong S_{2}^{e}$ by a complement $Q \cong S_{n}$ : each of the $e=\left(\begin{array}{l}n \\ k\end{array}\right) / 2$ direct factors $M^{\phi} \cong S_{2}$ of $M$ transposes the $k$-element sets $K$ and $N \backslash K$ in one equipartition $\phi=\{K, N \backslash K\}$ of $N$, while fixing all other elements of $\left(\begin{array}{l}N \\ k\end{array}\right)$; the complement $Q$ permutes $k$-element subsets through its natural action on $N$, and permutes the direct factors $M^{\phi}$ of $M$ by conjugation as it permutes the set $\Phi$ of all equipartitions $\phi$ of $N$. Let $\bar{G}$ denote the image of $G$ under the natural epimorphism 
Aut $J \rightarrow Q \cong S_{n}$; since $M$ acts trivially on $\Phi$, and $Q$ acts faithfully, one can regard $\bar{G}$ as the group induced by $G$ on $\Phi$. Since $G$ acts transitively on $\left(\begin{array}{l}N \\ k\end{array}\right), \bar{G}$ is transitive on $\Phi$, so $|\bar{G}|$ is divisible by $e$. Since $|G|=4 e$ it follows that $|G \cap M|$ divides 4 and $|\bar{G}|=e, 2 e$ or $4 e$.

As a subgroup of $S_{n}, \bar{G}$ has an action on $\left(\begin{array}{l}N \\ k\end{array}\right)$, agreeing with $G$ on $\Phi$. If $\bar{G}$ is transitive on $\left(\begin{array}{l}N \\ k\end{array}\right)$ then $\bar{G}$ is $k$-homogeneous on $N$, though not $k$-transitive since $|G|$ is not divisible by $\left(\begin{array}{l}n \\ k\end{array}\right) k$ !. However, Theorem 3.1 shows that no such group of degree $n$ exists for $k=n / 2$, so $\bar{G}$ is intransitive on $\left(\begin{array}{l}N \\ k\end{array}\right)$. Since it is transitive on $\Phi$ it must have two orbits on $\left(\begin{array}{l}N \\ k\end{array}\right)$, transposed by complementation.

If $|\bar{G}|=e$ or $2 e$ then $\bar{G}$ acts regularly or 2-regularly on its two orbits in $\left(\begin{array}{l}N \\ k\end{array}\right)$, so Lemma 7.2 implies that $n=4$ or 6 , a contradiction. Thus $|\bar{G}|=4 e=|G|$, so $\bar{G} \cong G$ and $|G \cap M|=$ 1. In this case $\bar{G}$ acts 4-regularly on its two orbits in $\left(\begin{array}{l}N \\ k\end{array}\right)$, so Lemma 7.2 implies that $n=10$ and $\bar{G}$ is the simple group $S:=P S L_{2}(8)=S L_{2}(8)$ of order 504, fixing a point $p \in N=\{1, \ldots, 10\}$ and acting naturally on $N \backslash\{p\}$, which is identified with $\mathbb{P}^{1}(8)$; its orbits on $\left(\begin{array}{l}N \\ k\end{array}\right)=\left(\begin{array}{l}N \\ 5\end{array}\right)$ consist of those 5-element subsets containing or not containing $p$, as in conclusion (3). (See Theorem 3.1(4) for the 4-homogenous action of $P S L_{2}(8)$ on $\mathbb{P}^{1}(8)$.)

We still need to demonstrate the existence of such 2-regular subgroups $G$ of Aut $J$, and here we will use some concepts from the cohomology of groups: the book [4] by Brown is an excellent reference.

The preceding argument shows that if $G$ exists, it is a complement for $M$ in the extension

$$
E:=M \rtimes S \leq M \rtimes Q=\text { Aut } J
$$

of $M$ by $S$. The conjugacy classes of such complements correspond to the elements of the first cohomology group $H^{1}(S, M)$ of $S$ with coefficients in $M$, which we will regard as an $\mathbb{F}_{2} S$-module [4, IV.2.3]: specifically, the class $[\gamma] \in H^{1}(S, M)$ containing a cocycle $\gamma: S \rightarrow M$ determines the conjugacy class containing a complement $G=\{\gamma(s) s \mid s \in S\}$. Now $M$ is the permutation module over $\mathbb{F}_{2}$ for $S$ acting on $\Phi$, or equivalently on the cosets of a Klein four-group $V=S_{\phi} \leq S$ stabilising some $\phi \in \Phi$. It follows that $M$ is the induced $\mathbb{F}_{2} S$-module $\operatorname{Ind}_{V}^{S} M^{\phi}$, where the corresponding direct factor $M^{\phi}\left(\cong \mathbb{F}_{2}\right)$ of $M$ is regarded as a trivial 1-dimensional $\mathbb{F}_{2} V$-module (see [4, III.5.5(a)], with coefficients reduced mod (2)). It therefore follows from Shapiro's Lemma [4, III.6.2 and III.5.9] that restriction from $S$ to $V$ and induction from $V$ to $S$ induce mutually inverse isomorphisms

$$
H^{1}(S, M) \cong H^{1}\left(V, M^{\phi}\right) .
$$

Since $V$ acts trivially on $M^{\phi}$ we have

$$
H^{1}\left(V, M^{\phi}\right) \cong \operatorname{Hom}\left(V, \mathbb{F}_{2}\right)=V^{*} \cong V
$$

[4. III.1, Exercise 2], so there are four conjugacy classes of complements $G$ for $M$ in $E$, with the conjugates of $S$ (which we will call the standard complements) corresponding to the zero cocycle. 
We now need to determine which of these complements act transitively on $\left(\begin{array}{l}N \\ 5\end{array}\right)$. Any complement $G$ for $M$ is transitive on $\Phi$, so it is either transitive or intransitive on $\left(\begin{array}{c}N \\ 5\end{array}\right)$ as the stabiliser $G_{K}=G \cap E_{K}$ in $G$ of some $K \in\left(\begin{array}{c}N \\ 5\end{array}\right)$ has index $\left|G: G_{K}\right|=252$ or 126 , or equivalently $G_{K}$ has order 2 or 4 . Now the orbit of $M$ containing $K$ consists of $K$ and $N \backslash K$, which are in different orbits of $S$, so $E_{K}=M_{K} \rtimes S_{K}$, where $M_{K}$ and $S_{K}$ are the stabilisers of $K$ in $M$ and $S$. Here $S_{K}$ is the Klein four-group $V=S_{\phi}$ stabilising $\phi=\{K, N \backslash K\} \in \Phi$, while $M_{K}=\oplus_{\psi \neq \phi} M^{\psi} \cong S_{2}^{125}$, with its direct factors generated by the transpositions corresponding to the equipartitions $\psi \in \Phi \backslash\{\phi\}$ of $N$. If we regard $M$ as the set of all functions $\Phi \rightarrow \mathbb{F}_{2}$, then $M_{K}$ consists of those sending $\phi$ to 0 .

Given a complement $G$ and a corresponding cocycle $\gamma: S \rightarrow M$, each element $g \in G$ has the form $g=\gamma(s) s$ for some unique $s \in S$. In particular, we have seen that $g \in G_{K}$ if and only if $s \in S_{K}$ and $\gamma(s) \in M_{K}$, or equivalently, $s \in S_{K}$ and $\gamma(s)$, regarded as a function $\Phi \rightarrow \mathbb{F}_{2}$, sends $\phi$ to 0 . Now $\gamma$ is induced from a cocycle $\delta: V \rightarrow M^{\phi}$, that is, a homomorphism $V \rightarrow \mathbb{F}_{2}$, with $\gamma=0$ if and only if $\delta=0$. Thus $\left|G_{K}\right|=|V \cap \operatorname{ker} \delta|=4$ or 2 as $G$ is standard or nonstandard. This confirms that the standard complements are intransitive on $J$, and shows that the three classes of nonstandard complements are transitive, as in conclusion (3).

(d) Finally, to deal with the case $k=(n-1) / 2$ we return to the main line of the proof, again following that of Theorem 1.1. If $I \neq k+1-I$ then Aut $J=S_{n}$ as in part (a), so we may assume that $I=k+1-I$. Thus Aut $J=S_{n+1}$, with its natural action on $N^{*}=N \cup\{n+1\}$ inducing actions on the set of equipartitions of $N^{*}$ and hence on $\left(\begin{array}{l}N \\ k\end{array}\right)$. Any 2-regular action of $G$ on $\left(\begin{array}{l}N \\ k\end{array}\right)$ corresponds to a 2-regular action on equipartitions, which amounts to an action on $N^{*}$ which is either regular on $\left(\begin{array}{c}N^{*} \\ k+1\end{array}\right)$ or has two 2-regular orbits on $\left(\begin{array}{c}N^{*} \\ k+1\end{array}\right)$ transposed by complementation. In the first case, $G$ is a $(k+1)$-homogenous but not $(k+1)$-transitive group of degree $n+1=2(k+1)$ on $N^{*}$, contradicting Theorem 3.1. In the second case Lemma 7.2 shows that $n=5$ and $k=2$, so the condition $I=k+1-I$ forces $I=\{1,2\}$, against our assumption that $I \neq\{1, \ldots, k\}$.

Remark. It is straightforward to check that in each of conclusions (1) and (2) there is a unique conjugacy class of 2-regular subgroups $G$ in Aut $J$. The following argument shows that the same applies to (3).

The action of $S$ on $\mathbb{P}^{1}\left(\mathbb{F}_{8}\right)$ extends naturally to $\tilde{S}:=P \Gamma L_{2}(8)$ (see Theorem $[3.1(4)$ ). This is a semidirect product of $S$ by the Galois group $\Gamma:=\mathrm{Gal} \mathbb{F}_{8} / \mathbb{F}_{2}$ of $\mathbb{F}_{8}$, a cyclic group of order 3 generated by the Frobenius automorphism $a \mapsto a^{2}$. We can take $\mathbb{F}_{8}=\mathbb{F}_{2}(t)$ where $t^{3}+t+1=0$. The Sylow 2-subgroup of the stabiliser $S_{\infty}=A G L_{1}(8)$ of $\infty$ in $S$ is invariant under $\Gamma$, as is its translation subgroup $V=\left\{0, t, t^{2}, t^{4}\right\} \cong V_{4}$ (note that $t^{4}+t^{2}+t=0$ ). The subgroup $\tilde{V}=V \rtimes \Gamma \cong A_{4}$ is the stabiliser in $\tilde{S}$ of the 4-element subset $V \subset \mathbb{P}^{1}\left(\mathbb{F}_{8}\right)$, so $M$ is the permutation module for $\tilde{S}$ on the cosets of $\tilde{V}$. The conjugacy classes of complements for $M$ in $\tilde{E}:=M \rtimes \tilde{S}$ therefore correspond to the elements of $H^{1}(\tilde{S}, M)$. As before, 
Shapiro's Lemma gives

$$
H^{1}(\tilde{S}, M) \cong H^{1}(\tilde{V}, N) \cong \operatorname{Hom}(\tilde{V}, N) .
$$

In this case, since $(\tilde{V})^{\text {ab }} \cong C_{3}$ we have $\operatorname{Hom}(\tilde{V}, N)=0$, so the only complements for $M$ in $\tilde{E}$ are the conjugates of $\tilde{S}$. This implies that none of the nonstandard complements $G$ for $M$ in $E$ is invariant under $\Gamma$, since otherwise it would yield a complement $G \rtimes \Gamma$ in $\tilde{E}$. Now $\Gamma$ permutes the three conjugacy classes of nonstandard complements, either trivially or transitively. Since $|\Gamma|=3$, whereas each conjugacy class has even order, it follows that $\Gamma$ cannot leave any class invariant. Thus the three conjugacy classes of 2-regular subgroups $G \leq E$ are equivalent under $\Gamma$, so they form a single conjugacy class in $\tilde{E}$. Since the choice of a subgroup $S \cong P S L_{2}(8)$ in $S_{10}$ is unique up to conjugacy, it follows that there is a single conjugacy class of 2-regular subgroups $G$ in Aut $J$.

\section{Appendix: NEAR-FIELdS AND SHARPly 2-TRANSITIVE Groups}

The following summary of near-fields and their connection with sharply 2-transitive permutation groups is adapted to suit our purposes from those given by Cameron in [6, §1.12], by Dixon and Mortimer in [10, §7.6], and by Hall in [14, §20.7].

A near-field $F$ satisfies all the usual field axioms, apart from possibly commutativity of multiplication $a b=b a$ and the left distributive axiom $a(b+c)=a b+a c$. (This concept should not be confused with that of a skew field, or division ring, in which only commutativity of multiplication is relaxed.) A near-field is a field if and only if multiplication is commutative.

In the finite case, near-fields are essentially equivalent to sharply 2-transitive permutation groups. Firstly, if $F$ is any near-field then the affine group

$$
A G L_{1}(F)=\{t \mapsto t a+b \mid a, b \in F, a \neq 0\}
$$

is a sharply 2-transitive group of transformations of $F$. The translations $t \mapsto t+b$ form a regular normal subgroup $N$, isomorphic to the additive group of $F$, while the transformations $t \mapsto t a(a \neq 0)$ form a complement, the stabiliser of 0 , isomorphic to the multiplicative group $F^{*}=F \backslash\{0\}$. Conversely, any sharply 2-transitive permutation group $G$ is a primitive Frobenius group, so if it is finite then it has an elementary abelian regular normal subgroup $N$ (the Frobenius kernel), which can be identified with the set permuted. Then the group structures of $N$ and of the stabiliser $G_{0}$ of the zero element $0 \in N$ provide the additive and multiplicative groups of a near-field $F$ with underlying set $N$, such that $G$ acts on $F$ as $A G L_{1}(F)$. Thus the classification of sharply 2-transitive finite groups is equivalent to that of finite near-fields; these classifications were achieved by Zassenhaus in [26, 27].

All but seven of the finite near-fields arise from a construction due to Dickson [9], in which the multiplicative group of a finite field is 'twisted' by field automorphisms to produce a non-commutative multiplication. Here, reversing the order used in [6, §1.12] and [10, §7.6], we will describe first the groups and then the near-fields. 
If $n$ is a prime power $p^{e}$ then for each $d$ dividing $e$ the field $\mathbb{F}_{n}$ has a (unique, cyclic) group of automorphisms $\Gamma$ of order $d$, generated by $\theta: t \mapsto t^{q}$ where $q=p^{e / d}$. The fixed field of $\Gamma$ is the subfield $\mathbb{F}_{q}$. Similarly, if

(a) $d$ divides $n-1=q^{d}-1$,

then $\mathbb{F}_{n}^{*}$ has a (unique, cyclic) subgroup $A$ of index $d$, consisting of its $d$ th powers. We will use these two groups to form a group $H$ of semilinear transformations of $\mathbb{F}_{n}$, fixing 0 and acting regularly on $\mathbb{F}_{n}^{*}$, so that we have a sharply 2-transitive group $G=N \rtimes G_{0} \leq$ $A \Gamma L_{1}\left(\mathbb{F}_{n}\right)$ of degree $n$, where $N$ is the translation group and $G_{0}=H$.

Suppose that

(b) $\{m(i) \mid i=0, \ldots, d-1\}$ is a complete set of residues $\bmod (d)$ in $\mathbb{Z}$,

so that if $\omega$ is any generator for the cyclic group $\mathbb{F}_{n}^{*}$ then $\left\{\omega^{m(i)} \mid i=0, \ldots, d-1\right\}$ is a set of coset representatives for $A$ in $\mathbb{F}_{n}^{*}$. We now let each $g \in A \omega^{m(i)} \subseteq \mathbb{F}_{n}^{*}$ induce the semilinear transformation

$$
\tau_{g}: t \mapsto t^{\theta^{i}} g=t^{q^{i}} g
$$

of $\mathbb{F}_{n}$, and we define $H:=\left\{\tau_{g} \mid g \in \mathbb{F}_{n}^{*}\right\}$. We need to ensure that $H$ is a group under composition. If $h \in A \omega^{m(j)}$ then (composing from left to right) we have

$$
\tau_{g} \circ \tau_{h}: t \mapsto\left(t^{q^{i}} g\right)^{q^{j}} h=t^{q^{i+j}} g^{q^{j}} h,
$$

so for this to have the form $\tau_{k}$ for some $k \in \mathbb{F}_{n}^{*}$ we must ensure that $g^{q^{j}} h \in A \omega^{m(i+j)}$. This will happen if our chosen residues $m(i)$ satisfy

$$
m(i+j)=q^{j} m(i)+m(j)
$$

for all $i$ and $j$. An obvious solution for this identity is to take each

$$
m(i)=1+q+q^{2}+\cdots+q^{i-1}=\frac{q^{i}-1}{q-1} .
$$

Then $H$ is closed under composition, so it is a subgroup of $\Gamma L_{1}\left(\mathbb{F}_{n}\right)$. It fixes 0 , and is transitive on $\mathbb{F}_{n}^{*}$ since $\tau_{g}$ sends 1 to $g$ for each $g \in \mathbb{F}_{n}^{*}$. Since $|H|=\left|\mathbb{F}_{n}^{*}\right|$ we deduce that $H$ acts regularly on $\mathbb{F}_{n}^{*}$, so that

$$
G:=\left\{t \mapsto t \tau_{g}+b \mid g \in \mathbb{F}_{n}^{*}, b \in \mathbb{F}_{n}\right\}
$$

acts on $\mathbb{F}_{n}$ as a sharply 2-transitive subgroup of $A \Gamma L_{1}\left(\mathbb{F}_{n}\right)$.

Note there is an epimorphism $H \rightarrow \Gamma, g \mapsto \theta^{i}$ where $g \in A \omega^{m(i)}$. The kernel is $A$, so (like $\left.\mathbb{F}_{n}^{*}\right) H$ is an extension of a normal subgroup $A$ by $\Gamma$. Indeed, $H$ and $\mathbb{F}_{n}^{*}$ induce the same group structure on their subgroup $A$, and also on its quotient group, even though $H$, being nonabelian, is not isomorphic to $\mathbb{F}_{n}^{*}$ if $d>1$.

Having motivated this definition of $m(i)$, we need to choose $q$ and $d$ carefully so that conditions (a) and (b) are satisfied. Elementary number theory (see [21, Theorem 6.4] or [27] for details) shows that the following is a sufficient condition for this:

(c) if $r$ divides $d$, where $r$ is prime or $r=4$, then $r$ divides $q-1$. 
The near-field $F$ corresponding to $G$ has the same underlying set and additive structure as $\mathbb{F}_{n}$, but multiplication (of non-zero elements $g$ and $h$ ) reflects composition of the corresponding elements of $\tau_{g}, \tau_{h} \in H$, so that the product of $g$ and $h$ in $F$ is given by $g \circ h=k=g^{q^{j}} h$ where $h \in A \omega^{m(j)}$ (and hence the centre of $F$ is $\mathbb{F}_{q}$ ). The group $G$ defined above can now be identified with the group $A G L_{1}(F)$ of affine transformations of this near-field $F$.

Dickson near-fields $F$ of order $n \equiv 3 \bmod (4)$ appear in Theorem 1.1(1). In such cases $e$ and hence $d$ are odd, so $H$ has a unique subgroup $H^{2}$ of index 2, consisting of those $\tau_{g}$ such that $g$ is a square in $\mathbb{F}_{n}^{*}$. Extending $H^{2}$ by the translation group gives the unique subgroup $A H L_{1}(F)$ of index 2 in $A G L_{1}(F)$. The involution $-1 \in \mathbb{F}_{n}^{*}$ is in $A$, so $\tau_{-1}$ acts on $F$ as $t \mapsto-t$; the involution $f: t \mapsto t \tau_{-1}+1$ in $A G L_{1}(F)$ thus preserves the 2-element subset $\{0,1\}$ of $F$, and hence generates its stabiliser. Since $n \equiv 3 \bmod (4),-1$ is a non-square in $\mathbb{F}_{n}^{*}$, so $A H L_{1}(F)$ does not contain $f$ and hence acts regularly on 2-element subsets of $F$.

In addition to the Dickson near-fields described above, there are seven exceptional finite near-fields $F$ : together with the Dickson near-fields, they appear in Theorem 1.2(1). They have order $n=p^{2}$, and thus correspond to sharply 2-transitive groups $G=A G L_{1}(F)$ of degree $n$, for the primes $p=5,7,11$ (twice), 23, 29 and 59. In each case, $G$ is a subgroup of $A G L_{2}(p)$ containing the translation group, and $G_{0}\left(\cong F^{*}\right)$ is a subgroup of $G L_{2}(p)$ acting regularly on non-zero vectors. When $p=5,7$ or 11 one can take $G_{0}$ to be the binary tetrahedral, binary octahedral or binary icosahedral group $2 T \cong S L_{2}(3), 2 O \cong 2 . S_{4}^{-}$or $2 I \cong S L_{2}(5)$; for $p=11$ (again), 23,29 or 59 one can take $G_{0}=2 T \times C_{5}, 2 O \times C_{11}, 2 I \times C_{7}$ or $2 I \times C_{29}$, with the cyclic direct factor consisting of scalar matrices.

\section{ACKNOWLEDGMENT}

The second author acknowledges the support by the projects VEGA 1/0577/14, VEGA 1/0474/15, NSFC 11371307, and both authors acknowledge support by the project Mobility - enhancing research, science and education at the Matej Bel University, ITMS code: 26110230082, under the Operational Program Education cofinanced by the European Social Fund.

\section{REFERENCES}

[1] R. A. Beaumont and R. P. Peterson, Set-transitive permutation groups, Canad. J. Math. 7 (1955), $35-42$.

[2] N. L. Biggs, Automorphisms of imbedded graphs, J. Combin. Theory Ser. B 11 (1971), 132-138.

[3] N. L. Biggs, Some odd graph theory, Second International Conference on Combinatorial Mathematics (New York, 1978), pp. 71-81, Ann. New York Acad. Sci., 319, New York Acad. Sci., New York, 1979.

[4] K. S. Brown, Cohomology of Groups, Springer, 1982.

[5] P. J. Cameron, Transitivity of permutation groups on unordered sets, Math. Z. 148 (1976), 127-139.

[6] P. J. Cameron, Permutation Groups, London Math. Soc. Student Texts 45, Cambridge University Press, Cambridge, 1999.

[7] P. J. Cameron, P. M. Neumann and D. N. Teague, On the degrees of primitive permutation groups, Math. Z. 180 (1982), 141-149. 
[8] J. H. Conway, R. T. Curtis, S. P. Norton, R. A. Parker and R. A. Wilson, ATLAS of Finite Groups, Clarendon Press, Oxford, 1985.

[9] L. E. Dickson, On finite algebras, Nachr. Akad. Wiss. Göttingen, Math.-Phys. Kl. II (1905), 358-393.

[10] J. D. Dixon and B. Mortimer, Permutation Groups, Graduate Texts in Mathematics 163, Springer, 1996.

[11] E. Dobson and A. Malnič, Groups that are transitive on all partitions of a given shape, J. Algebraic Combin. 42 (2015), 605-617.

[12] G. Gauyacq, On quasi-Cayley graphs, Discrete Appl. Math. 77 (1997), no. 1, 43-58.

[13] C. D. Godsil, More odd graph theory, Discrete Math. 32 (1980), 205-207.

[14] M. Hall, Jr, The Theory of Groups, Macmillan, New York, 1959.

[15] R. Jajcay and G. A. Jones, $r$-regular families of graph automophisms, in preparation.

[16] R. Jajcay, A. Malnič, and D. Marušič, Counting closed oriented walks in vertex-transitive graphs, Discrete Math. 307, 3-5 (2007), 484-493.

[17] G. A. Jones, Automorphisms and regular embeddings of merged Johnson graphs, European J. Combin. 26 (2000), 417-435.

[18] W. M. Kantor, Automorphism groups of designs, Math. Z. 109 (1969), 246-252.

[19] W. M. Kantor, $k$-homogeneous groups, Math. Z. 124 (1972), 261-265.

[20] D. Livingstone and A. Wagner, Transitivity of finite permutation groups on unordered sets, Math. Z. 90 (1965), 393-403.

[21] H. Lüneburg, Ein einfacher Beweis für den Satz von Zsigmondy über primitive Primteiler von $a^{N}-1$, in Geometries and Groups, Lecture Notes in Math. 893, Springer-Verlag, Berlin, 1981, pp. 219-222.

[22] B. D. McKay and C. E. Praeger, Vertex-transitive graphs which are not Cayley graphs, I, J. Austral. Math. Soc., Ser. A 56 (1994), 53-63.

[23] P. Potočnik, P. Spiga and G. Verret, Cubic vertex-transitive graphs on up to 1280 vertices, J. Symbolic Computation 50 (2013), 465-477. Associated data available at http://www.matapp.unimib.it/ spiga/census.html.

[24] G. Sabidussi, Vertex-transitive graphs, Monatsh. Math. 68 (1964), 426-438.

[25] R. A. Wilson, The Finite Simple Groups, Springer, 2009.

[26] H. Zassenhaus, Kennzeichnung endlicher linearer Gruppen als Permutationsgruppen, Abh. Math. Sem. Hamburg 11 (1936), 17-40.

[27] H. Zassenhaus, Über endliche Fastkörper, Abh. Math. Sem. Hamburg 11 (1936), 187-220.

Gareth A. Jones, Mathematical Sciences, University of Southampton, Southampton, United Kingdom AND Institute of Mathematics and Computer Science, Matej Bel University, Banská Bystrica, Slovakia

E-mail address: G.A.Jones@maths.soton.ac.uk

ROBERT JAJCAY,

Comenius University, Bratislava, Slovakia, AND

University of Primorska, Koper, Slovenia

E-mail address: robert.jajcay@fmph.uniba.sk 